\title{
The Th1/Th2/Th17 cytokine profile of HIV-infected individuals: a multivariate cytokinomics approach
}

\author{
Williams Aurelia ${ }^{a}$, Steffens Francois ${ }^{b}$, Reinecke Carolus ${ }^{c}$ and Meyer Debra ${ }^{a *}$ \\ ${ }^{1}$ Department of Biochemistry, University of Pretoria, Pretoria 0002, South Africa \\ ${ }^{b}$ Department of Statistics, University of Pretoria, Pretoria 0002, South Africa \\ ${ }^{c}$ Centre for Human Metabonomics, North-West University, Potchefstroom Campus, Potchefstroom \\ 2520 , South Africa \\ * Author to whom correspondence should be addressed: \\ Tel: $\quad$ +27 124202300 \\ Fax: $\quad+27123625302$ \\ debra.meyer@up.ac.za
}

${ }^{1}$ Abbreviations

\begin{tabular}{ll}
\hline${ }^{1}$ ART & Antiretroviral Therapy \\
CBA & Cytometric Bead Array \\
Th & T Helper cell \\
LDA & Linear Discriminant Analysis \\
IL & Interleukin \\
ANOVA & Analysis of Variance \\
ELISA & Enzyme Linked Immunosorbent Assay \\
MFI & Mean Fluorescence Intensity \\
PCA & Principal Component Analysis \\
NMR & Nuclear Magnetic Resonance
\end{tabular}




\section{Abstract}

HIV infection causes the dysregulation of cytokine production. A cytokinomics approach employing cytometric bead array (CBA) technology, flow cytometry and multivariate analysis was applied to the investigation of HIV-induced T helper cell type 1 (Th1), Th2 and Th17 cytokine changes in the serum of treatment naive individuals. Stepwise linear discriminant analysis (LDA) and logistic regression identified interleukin (IL)-6 to be discriminatory for HIV infection with 74.6 and $71.2 \%$ of the cases correctly classified. Analysis of variance (ANOVA) confirmed IL-6 and IL-10 concentrations to be significantly $(p=0.001$ and $p=0.025)$ different between the groups. A scatter plot of the log IL-6 and IL-10 concentrations for the groups largely overlapped, with improved differentiation where patients were advancing to the acquired immunodeficiency syndrome (AIDS). IL-17A levels were higher than other cytokines but did not significantly distinguish the groups suggesting that the HIV-and HIV+ individuals had similar immune profiles. This possibility was supported by other clinical indicators. Taken together, the measured cytokines (IL-6, 10 and 17) have potential prognostic value.

Keywords: Cytometric Bead Array - Flow cytometry - Cytokinomics • Interleukin • Multivariate

\section{Introduction}

HIV infection disrupts cytokine production and function of the CD4+ T helper and CD8+ cytotoxic T cells with disease progression mainly associated with a Th2 cytokine profile despite some contradictory references [1-3]. Tumor necrosis factor alpha (TNF- $\alpha$ ), interferon gamma (IFN- $\gamma$ ), IL-1, IL-2, , , IL-6 and IL-10 are some of the cytokines affected during HIV infection. Measuring these cytokines gives an indication of the degree of immune activation (elevated levels of TNF- $\alpha, \mathrm{IL}-6$ and IL-1), the extent of the immune response and disease progression. 
A newly defined subset of CD4 cells [4] called Th 17 cells produce, amongst others, the pro-inflammatory IL-17A [5]. Th17 cells and cytokines are associated with protection against bacterial, fungal and viral infections $[4,6]$ and are important for maintaining a healthy mucosa. Because HIV primarily infects CD4 cells (including the Th17 subset), infection could potentially lead to a decrease in IL-17A production [5 and reviewed in 7] leaving the affected host susceptible to opportunistic infections $[5,8]$. The role of Th17 cells and cytokines in the context of HIV infection is not entirely resolved but their possible involvement as prognostic indicators, contributors to disease progression and/or as antiviral agents has been reported. Generally, Th17 cells and cytokines are elevated during early infection [4, 9] when viral load is high but is lowered during chronic infection or in long term-non progressors [9] when viral load is low. Measuring IL-17A secretion in conjunction with other cytokines could shed light on whether this protein is influenced by or can influence other cytokine groups and also whether the behaviour of this cytokine is in line with the Th2 rather than Th1 cytokine groups during HIV-1 infection.

With the advent of faster analysis times and the possibility of obtaining more information from limited sample volumes, the potential clinical value of measuring multiple cytokines to probe immune dysfunction and immune-based diseases has gained popularity $[6,10]$. Measuring cytokine alterations during HIV infection [11-16] and other pathological states [17-21] is increasingly being investigated for use in predicting disease progression. Most studies have however used univariate statistical and correlation analyses. In an investigation by Roberts et al [15], Cox proportional hazards regression was used to show that the plasma cytokine profile can predict disease progression in an acute model of HIV infection. Kamat et al [11] applied both uni- and multivariate approaches and identified a plasma cytokine signature capable of distinguishing samples collected from HIV patients (on treatment) from uninfected controls.

While IL-17A has mainly been measured following the isolation and stimulation of PBMCs in vitro, this study measured secreted cytokine levels following natural infection. 
Multiplexing technologies allowed the analysis of the Th1/Th2/Th17 cytokinome through flow cytometric detection, while multivariate analysis identified specific cytokines (affected by HIV) which were also able to distinguish between the experimental groups. Cytokinomics first coined by Clerici (2010) [22] is used here in the tradition of other 'omics' approaches to mean the measurement and statistical evaluation of the cytokinome of a particular system.

The effect of HIV on the Th1/Th2/Th17 cytokine profile using multivariate approaches, as is done in this study on the serum of clinically stable patients has not been investigated before. The ability of this group of molecules to distinguish between uninfected and infected cases has not been investigated either. The detection of a number of secreted cytokines and the application of multivariate statistics to extract information on their interactions as influenced by HIV infection, with commentary on the potential role of these molecules as biomarkers of HIVIAIDS is also presented here.

Th1/Th2/Th17 cytokines were thus collectively assayed to evaluate the potential of this group of cytokines as markers of HIV infection and disease progression in this patient group. LDA and logistic regression identified IL-6 while ANOVA identified IL-6 and IL-10 to be discriminatory for HIV infection. Using the log IL-6 and IL-10 concentrations as discriminants, a scatter plot was shown to overlap for healthier individuals but separated with disease progression. IL-17A was detected in both experimental groups and associated with the clinically stable state of the majority of the patients. Following the inclusion of patients progressing to AIDS, IL-17A was marginally lowered in the HIV+ group. Collectively, the Th1/Th2/Th17 cytokines when assessed in relation to each other serve as potential prognostic indicators in the absence of antiretroviral therapy (ART). 


\section{Materials and Methods}

\subsection{Ethics Approval}

Ethics approval was obtained from the Faculties of Natural and Agricultural Sciences and Health Sciences of the University of Pretoria (protocols E080-506-019 and 163/2008 respectively).

\subsection{Sample preparation}

Blood samples of HIV seropositive donors $(n=25)$ were obtained from the Steve Biko Academic Hospital in Pretoria and King's Hope Development Foundation Clinic in Diepsloot, Johannesburg. The participating donors were HIV positive, had not been diagnosed as having AIDS (CD4 counts above 200 cells/ $\mu$ l blood) and were not on ART. In a few cases, CD4 counts of $<200$ cells $/ \mu$ l were detected after the study was initiated. These cases were retained since they had not commenced ART. Viral load measurements were determined by means of the COBAS AmpliPrep/COBAS TaqMan HIV-1 Test (Roche Molecular Systems, Inc Pleasanton, CA). This parameter was not part of the inclusion criteria since practices at the source clinics were such that the viral load of individuals was determined only when donors were tested for HIV infection for the first time and immediately before treatment was initiated. HIV negative donors $(n=34)$ had no known metabolic or other medical condition at the time of blood collection and their HIV negative status confirmed with the VISITECT ${ }^{\circledR}$ HIV $1 / 2$ rapid tests (Omega Diagnostics Limited, Scotland, UK). The samples were reasonably well-matched in terms of gender but not age. Additional information relating to the participating donors is summarized in Table 1.

Venous blood was collected in non-EDTA vacutainers (Greiner Bio-One $\mathrm{GmbH}$, Kremsmünster). Serum was removed after centrifugation at $1610 \times \mathrm{g}$ for 10 minutes and stored at $-70^{\circ} \mathrm{C}$ until use. 
Table 1. Characteristics of the participating donors.

\section{HIV Status}

\begin{tabular}{|c|c|c|}
\hline Females (\%) & 58.8 & 52.0 \\
\hline Males (\%) & 41.2 & 48.0 \\
\hline Mean age \pm SD (years) & $23.71 \pm 5.17$ & $37.75 \pm 11.09$ \\
\hline Mean CD4 count (cells/ $\mu$ l blood) & Not applicable & $323.46 \pm 135.72$ \\
\hline Viral load (copies/ml, range) & Not applicable & 1030359.5 (2328-8 841057$)$ \\
\hline
\end{tabular}

\subsection{Cytometric Bead Array (CBA)}

Cytokine analysis was done using the Human Th1/Th2/Th17 CBA kit (BD Biosciences, San Jose, California) which allowed for the simultaneous detection of IL-2, IL-4, IL-6, IL-10, TNF- $\alpha$, IFN- $y$ and IL-17A. Aliquots of sera were thawed, diluted with assay diluent $(1: 2 \mathrm{v} / \mathrm{v})$ and CBA analysis performed as per the manufacturer's instructions. Cytokine standards were serially diluted to facilitate the construction of calibration curves necessary for determining protein concentrations of test samples. Beads coated with capture antibodies specific to the above named cytokines, were mixed. Fifty microliters of the capture bead mixture was added to $50 \mu \mathrm{l}$ of sample. To this sample-bead mixture, $50 \mu \mathrm{l}$ of Phycoerythrin-conjugated detection antibody was added. The mixture was incubated for three hours in the dark with occasional shaking to allow sandwich complexes to form which yield a fluorescent signal that is proportional to the concentration of the respective analyte. The samples were washed with $1 \mathrm{ml}$ of wash buffer $(200 \times \mathrm{g}, 5$ minutes $)$ and the pellet resuspended in $300 \mu \mathrm{l}$ wash buffer. Two hundred microlitres of each sample was plated on PRO-BIND ${ }^{\mathrm{TM}} 96$ well assay plates and analyzed on the FACSArray Bioanalyzer using FCAP FCS Filter and FCAP Array Software (BD Biosciences, San Jose, CA, USA). Using these software packages, debri was filtered from the data and identification of the bead populations and their mean fluorescence intensities (MFls) automated. Each cytokine bead was measured $\sim 200$ times before the MFI was averaged. The MFI of test samples were 
then fitted into the 5-parameter logistic curve-fitting equation to obtain the concentration of cytokines in test samples which finally incorporated the dilution factor. Three separate experiments were performed for every sample. To ensure accuracy/validity of the data, cytokine levels were measured following calibration of the instrument with SPHERO 8-peak Rainbow Particles. These particles are of uniform size but differ in fluorescence intensity and allow for the calibration of all channels of the flow cytometer. These particles are especially important for checking the sensitivity and resolution of the instrument. In addition cytokine standards of known concentration were run alongside the samples to allow for quantitation. The CBA kit itself has decreased handling steps which translates into less handling errors. Using a commercial kit such as this with internal standards helped with standardizing the data to an extent.

\section{Statistical Analysis}

\subsection{Standardization of the data}

Statistical analysis was performed using IBM SPSS (version 19.0). The Cronbach alpha was calculated to judge internal consistency. The calculated value was alpha $=0.708$ which is reasonable for such a diverse sample group. Cytokine concentrations of each sample were averaged and a pooled average of the HIV-and HIV+ samples log transformed to make the scales of the data more comparable.

\subsection{Linear Discriminant Analysis (LDA)}

Using the log-transformed cytokine concentrations, LDA was applied to classify the cases as HIV- or HIV+. Stepwise discriminant analysis was used to select the best set of cytokines for the classification during which IL-6 was identified as the best discriminatory parameter of infection. 


\subsection{Logistic Regression}

Logistic regression is a model for the probability that an individual case belongs to a particular group. A stepwise analysis was used to select the cytokines which would best classify the two groups. IL-6 was again identified as the best parameter.

\subsection{Analysis of Variance (ANOVA)}

Comparison of the mean log-transformed cytokine concentrations of the HIV-and $\mathrm{HIV}+$ cases was done by means of analysis of variance F-tests to establish whether or not there were significant differences between the groups. Only cases where the significance level ( $p$-value) was less than 0.05 were considered. IL-6 and IL-10 levels were significantly different between the HIV- and HIV+ groups while IL-17A was detected in both experimental groups. The latter cytokine's levels were marginally lower in the HIV+ group.

\section{Results and Discussion}

The simultaneous analysis of multiple cytokines in one experiment was not extensively done prior to technological advances such as CBA analysis. Chemometrics which employs multivariate statistical approaches has become valuable in simplifying the analysis and interpretation of large data sets. In this study, changes in the serum Th1/Th2/Th17 cytokine profile of clinically stable ART naive individuals in the asymptomatic chronic phase of HIV infection was investigated using CBA technology and multivariate statistics.

CBA kits are supplied with standard samples and allow quantitative information to be determined for test samples. Figure S1 (see supplementary data) shows representative examples of the standard curves obtained for each cytokine $\left(R^{2}\right.$ was $\geq 0.99$ for all the plots shown). The MFI of test samples were fitted into the 5-parameter logistic curve-fitting equation to obtain the corresponding cytokine concentrations which were standardized to allow for further statistical processing. Because cytokines form part of a network, the profile 
Table 2. Classification of experimental cases as HIV- or HIV+ using stepwise (i) linear discriminant analysis and (ii) logistic regression.

\section{Predicted group membership}

Group

HIV- HIV+ Total Percentage correct Percentage incorrect

(i) LDA classification results ${ }^{\mathrm{a}}$

$\begin{array}{llllll}\text { HIV- } & 31 & 3 & 34 & 91.2 & 8.8 \\ \text { HIV+ } & 12 & 13 & 25 & 52.0 & 48.0\end{array}$

Wilks' Lambda

Exact $F$

Step Entered Statistic df1 df2 df3 Statistic df1 df2 df3

Variables entered/removed ${ }^{c, a}$

$\begin{array}{llllllll}1 & \text { LNIL6 } & .819 & 1 & 1 & 57.000 & 12.615 & .001\end{array}$

Predicted group membership

Group

HIV- HIV+ Total Percentage correct Percentage incorrect

(ii) Logistic regression classification table ${ }^{\mathfrak{b}}$

$\begin{array}{rrrrrr}\text { HIV- } & 31 & 3 & 34 & 91.2 & 8.8 \\ \text { HIV+ } & 14 & 11 & 25 & 44.0 & 56.0 \\ & & & \text { B } & \text { S.E. Wald Df Sig. Exp (B) }\end{array}$

Variables in the equation ${ }^{c}$

Step $1^{\mathrm{a}}$ LNIL6 $\quad 1.476 \quad .5537 .1271 \quad .0084 .377$

Constant $-1.261 .4328 .5191 \quad .004 .283$

${ }^{\mathrm{a}} 74.6 \%$ of original grouped cases correctly classified.

${ }^{b} 71.2 \%$ of original grouped cases correctly classified. 
${ }^{\mathrm{C}}$ Variable(s) entered on step 1: LNIL6.

${ }^{d} \mathrm{~F}$ level, tolerance, or VIN insufficient for further computation.

obtained is better understood if all or most variables contributing to it are analyzed simultaneously. Variables may not be significant when analyzed in isolation but this may change when analyzed in combination with other variables. Multivariate statistics (LDA and logistic regression) where more than one variable is measured and analyzed at a time was therefore employed. Table 2 shows that LDA was able to correctly classify $74.6 \%$ of cases with IL-6 selected as the best discriminatory cytokine in relation to all other cytokines measured. Also shown is the percentage of misclassified cases; $9 \%$ (HIV-) and $48 \%$ $(\mathrm{HIV}+)$.

Table 2(ii)fig shows that logistic regression correctly classified $71.2 \%$ of the cases. IL-6 was once again identified as the discriminatory cytokine. Although the overall percentages of correctly classified cases are similar between LDA and logistic regression, these analyses misclassified a large number of $\mathrm{HIV}+$ cases (48 and $56 \%$ respectively). These misclassifications may be a consequence of the experimental groups presenting with closely related cytokine profiles. The HIV+ individuals thus present similar immunological profiles to the HIV- individuals making it difficult for LDA and logistic regression to distinguish between these cases with $100 \%$ accuracy. Patient records reflecting CD4 counts in particular identified these individuals as being infected but not diagnosed as having AIDS suggesting an apparently clinically stable health condition also supported by the measured cytokines.

ANOVA showed that IL-6 and IL-10 levels were dissimilar (visual representation in Figure 1) and differentiated the groups from one another ( $p=0.001$ and 0.025$)$. The log IL-6 and IL-10 concentrations of the two groups were plotted on a scatter diagram (Figure 2). Poor separation of the groups was evident and is expected because of the clinically stable condition of the majority of the patients (based on CD4 data) causing them to behave closer 


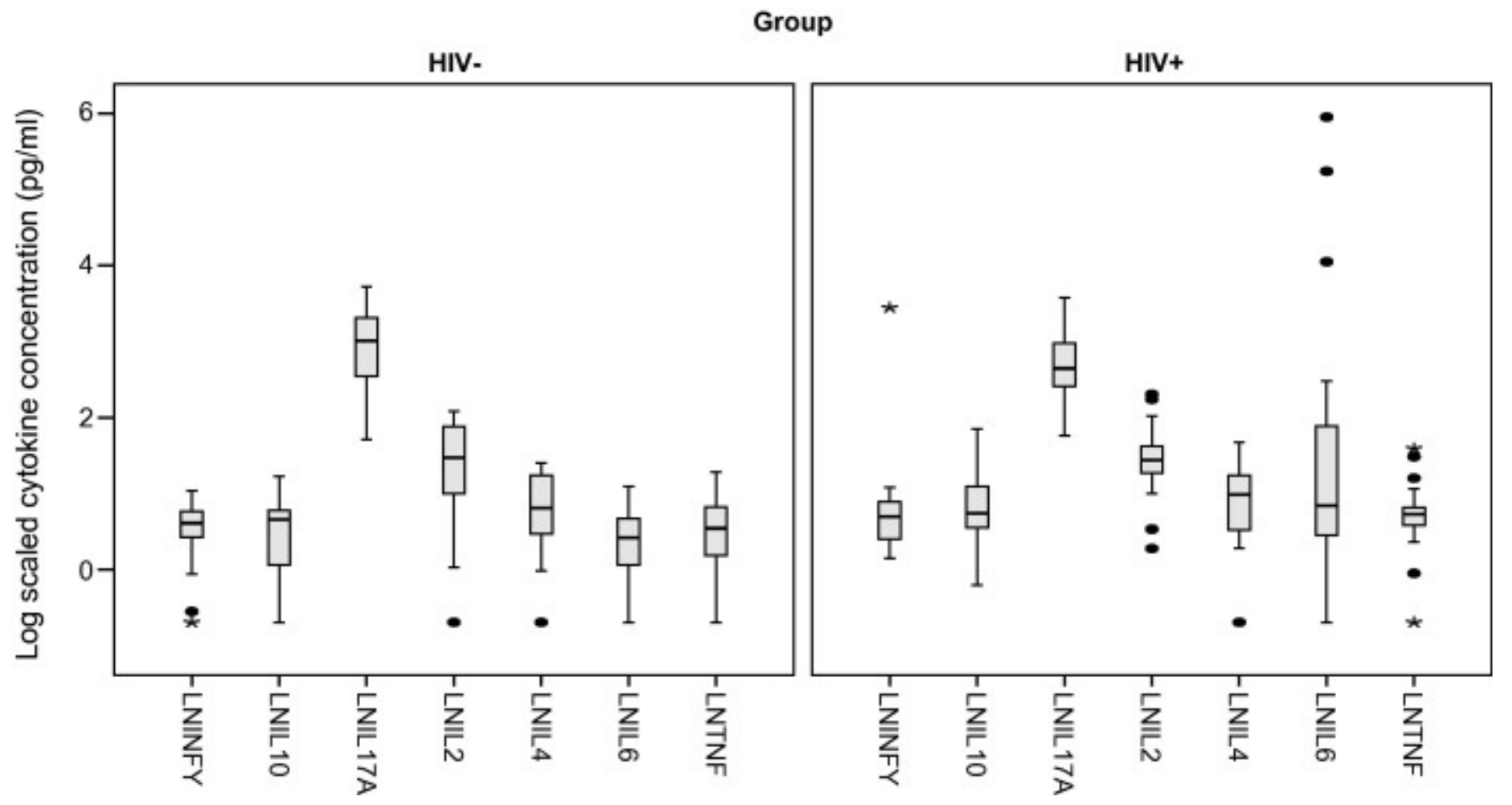

Fig. 1. Secreted Th1/Th2/Th17 cytokines in HIV- and HIV+ sera measured using the CBA kit (BD Biosciences) and flow cytometry. Cytokine concentrations were log scaled and statistical significance determined using ANOVA. IL-6 and IL-10 levels differed significantly between HIV- and HIV+ groups ( $p=0.001$ and 0.025$)$. IL-17A was detected in both groups but was marginally lower in the HIV group although this difference was not statistically significant.

to their uninfected counterparts. This may be explained by the fact that during the asymptomatic phase of infection, the infected individuals present with minimal phenotypic changes representative of HIV-induced immunological alterations. These results suggest the immunological profile of treatment naive, clinically stable individuals to not differ significantly from that of uninfected controls. The bivariate plot showed three cases to be clustered further away implying a more drastic alteration in their cytokine profile. Upon closer inspection, it was found that these were samples with CD4 counts $<200$ cells $/ \mu$ l blood. The viral load for the top most of the three samples was in excess of 8 million copies $/ \mathrm{ml}$ plasma. This suggests that as HIV infection progresses to AIDS (low CD4 counts and high viremia) 




Fig. 2. Scatter plot of log IL-6 and IL-10 concentrations showing an overlap between the groups. Separation of the groups improved (at LNIL-6 and LNIL-10>1) when cases representative of progression from HIV to AIDS were included (low CD4 counts and high viral loads).

the discriminatory power of cytokines are improved. These findings are supportive of the view that laboratory markers are more abnormal with advanced disease [23]. In the cohort of Kamat et al [11] samples from individuals with advanced disease (CD4 nadir $<300$ cells/ $\mu$ l blood) having variable viral loads due to the administration of ART or lack thereof, were assayed. These were correctly classified with $80 \%$ accuracy using unsupervised hierarchical clustering. In the present article supervised approaches were mainly used. Similar to our findings, the HIV+ cases of Kamat et al [11] when displayed on a PCA plot could not be effectively discriminated from the uninfected controls. Biomarker levels (particularly IL-6) were mostly elevated in the viremic patients. Because the patients were in the advanced stage of the disease, the authors suspect the $80 \%$ classification to be an overestimation. 
Using metabonomics, our group showed that HIV-, HIV+ and AIDS cases on ART could be distinguished from each other based on ${ }^{1} \mathrm{H}$ NMR metabolic profiles even though the viral load range was large [24]. Unlike Hewer et al's [24] metabolic data, the cytokine profile measured here was biased in terms of viral load, yielding clearer separation profiles where samples representative of disease progression were included. Given the limited number of cases, those having low CD4 counts were retained in the analyses as they complied with the selection criteria for this investigation (HIV+ and treatment naive). These samples therefore provided insight into how AIDS affected cytokine changes. Where those cases having low CD4 counts were removed from the analysis, the mean concentration of IL-6 and IL-10 was still discriminatory for HIV infection although with a lower degree of significance. Similar to Jansen et al [25], it seems that viral load is the driving force determining the extent of the immune response. Keating et al [12] also showed this influence of viral load on the extent of cytokine perturbation (cytokines were less altered when viremia was lowered through treatment).

The remaining cytokines (TNF- $\alpha$, INF- $\gamma$, IL-2, IL-4 and IL-17) resulted in a profile that was generally indistinguishable for the two groups. The levels of most of these cytokines were slightly more variable in the HIV+ compared to the control group. These cytokines being indistinguishable between the experimental groups is also supportive of a mostly clinically stable HIV+ cohort. IL-17A was elevated in concentration compared to all other cytokines measured (Figure 1). Although not significantly different in concentration between the groups, it was marginally lowered in the HIV+ group. Yue et al [9] documented IL-17A to be low or undetected in the blood of HIV- individuals. This finding is in contrast to data presented here where IL-17A was detected in uninfected individuals (Figure 1, left). In agreement with data presented here, Brenchley et al [26] detected Th17 cells and IL-17A in the peripheral blood (and gastrointestinal tract) of healthy uninfected individuals.

IL-17A is reported to be elevated soon after infection but lowered during chronic infection and in long term non-progressors [9]. That the IL-17A levels were similar between 
HIV- and HIV+ individuals could be supportive of the clinically stable state of the majority of patients (supported by CD4 counts). When patients progressing to AIDS were included, IL17A levels were lowered. Literature documenting an inverse correlation between viral load, the number of Th17 cells and therefore IL-17A exists [5]. In these articles IL-17A was measured following the isolation and in vitro stimulation of cells. The sera cytokines measured in the present study are in the context of natural infection and were measured ex vivo with no activation stimulus. Th17 cells can regulate the Th1 pathway [27] and therefore the production and secretion of IL-17A may signal antiviral responses. Because the patients used in this study were clinically stable, there was no in vivo stimulus for increased cytokine production and therefore levels detected in vitro appear similar to that of negative controls.

If considered collectively with the IL-6 and IL-10 concentrations, IL-17A levels allows for commentary on disease progression. Metabonomics studies routinely identifies several molecules which are indicative of disease progression and the distinction disappears if single molecules are considered in isolation. This was demonstrated by Williams et al [28] where a metabonomics approach was applied to the evaluation of the organic acid profile of treatment naive patients and showed several molecules to be indicative of disease progression. Because the immune and metabolic systems are linked, the metabonomics findings support the possibility that more than one cytokine should be considered for prognostic purposes.

IL-6, IL-10 and possibly IL-17A thus function as potential markers during HIV infection. IL-6 and IL-10 is representative of Th2 type cytokines and are usually associated with disease progression while low IL-17 levels have been linked to chronic infection and non-progression. The former two cytokines distinguished the infected and uninfected groups while IL-17 supported the clinically stable status of the patients suggesting that the levels of IL-6 and 10 measured here was not high enough to be indicators of disease progression for all but three samples but still indicate active infection. Elevated levels of IL-6 are associated with HIV infection as well as aging [14]. Although the mean age of the HIV+ individuals was 
higher than that of the uninfected control group (Table 1), age could not have served as a confounding factor since one of the HIV+ samples which clustered far away was from a younger individual (22 years of age).

In an acute model of HIV infection, Roberts et al [15] showed IL-6 and IL-10, amongst other cytokines, to be elevated. These authors also showed IL-10 to be correlated to viral load. In another South African-based study INF- $\gamma$, IL-6 and IL-10 were elevated in a group of HIV-infected individuals with immune reconstitution inflammatory syndrome [16]. IL2 and IL-6 were key cytokines elevated in simian immunodeficiency virus-infected monkeys with encephalitis [12]. These examples supports IL-6 and IL-10 levels as having a role in the immune response against HIV infection and potentially also in monitoring disease progression.

A limitation of this work however is that incomplete demographic and other patient information prevented the consideration of all potentially confounding variables. The data can however still be regarded as valid (validity here is interpreted as meaning the extent to which measurements were well-founded and accurately comparable to the actual situation during infection) since cytokines disclosed by this investigation proved to be sufficiently relevant and (in some cases) significantly (IL-6 and 10) different to be taken into account in studies on HIV infection. It is, however, noteworthy that the samples that were the most differentiated (Figure 2) had low CD4 counts and high viral loads. The top most sample had a viral load in excess of 8 million copies/ml plasma. This could mean that for the chronic phase of HIV infection cytokine changes are subtle and increase or become more distinct with disease progression. Whether this is a consistent characteristic of disease progression - from chronic HIV infection to full-blown AIDS -as similarly observed for our metabonomics findings [28] will have to be addressed by more comprehensive investigations.

To conduct controlled experiments on human samples is often impractical. Cytokine responses amongst individuals having different backgrounds will vary causing a spread in the data. Such moderate to large standard deviations are evident in Figure 1. It is also seen 
in a recent publication where the plasma cytokine profile of individuals was measured before and after infection [15], which is supposedly a more controlled infection model. For the data presented in this work the Cronbach alpha was calculated to judge internal consistency. The calculated value was alpha $=0.708$ which is reasonable for such a diverse sample group. Based on the inclusion of quality controls (instrument calibration, internal standards, non HIV-infected samples), the exclusion of one major confounding factor (age) and the significant cytokines detected (which correlates well with literature), the data presented in this article is considered valid and reliable.

In conclusion, the simultaneous assessment of many cytokines through multiplexing and multivariate analysis showed minimal changes in the cytokine profile of HIV+ individuals during the asymptomatic phase of infection. This changed with the progression of HIV infection to AIDS with cytokines of statistical significance being able to classify more than 70 $\%$ of the cases correctly. Cytokinomics in addition to quantifying cytokine levels in HIV-and HIV+ samples allowed for discrimination between the experimental groups. This approach discloses information on specific cytokines as potential markers of disease progression in a group of clinically stable patients prior to ART. There is however a need for studies with higher patient numbers and perhaps with viral load as the guiding parameter where the simultaneous analysis of multiple cytokines, be it different combinations of these molecules, is evaluated to further clarify the usefulness of these molecules as biomarkers for HIVIAIDS.

\section{Acknowledgements}

This work was supported by the National Research Foundation, Technology Innovation Agency, Medical Research Council and the Faculty of Natural and Agricultural Sciences at the University of Pretoria.

\section{Conflict of Interest}

The authors declare no conflict of interest. 


\section{References}

[1] Sarih M, Maâtaoui WE, Benslimane A. Analysis of apoptosis and a Th1/Th2 phenotype in HIV-infected patients. Immunol Lett 1996; 50:125-29.

[2] Graziosi C, Pantaleo G, Gantt KR, Fortin J, Demarest JF, Cohen OJ, et al. Lack of evidence for the dichotomy of $\mathrm{TH} 1$ and $\mathrm{TH} 2$ predominance in HIV-infected individuals. Science 1994; 265:248-52.

[3] Maggi E, Mazzetti M, Ravina A, Annunziato F, Carli MD, Piccinni MP, et al. Ability of HIV to promote a $\mathrm{TH} 1$ to $\mathrm{THO}$ shift and to replicate preferentially in $\mathrm{TH} 2$ and $\mathrm{THO}$ cells. Science 1994; 265:244-48.

[4] van de Veerdonk FL, Gresnigt MS, Kullberg BJ, van der Meer JW, Joosten LA, Netea MG. Th17 responses and host defense against microorganisms: An overview. BMB Rep 2009; 42:776-87.

[5] Ndhlovu LC, Chapman JM, Jha AR, Snyder-Cappione JE, Pagan M, Leal FE, et al. Suppression of HIV-1 plasma viral load below detection preserves IL-17 producing T cells in HIV-1 infection. AIDS 2008; 22:990-92.

[6] Salem ML, Alenzi FQ, Nath N, El-Naggar S, Al-Khami A, Al-Jabri A, et al. Plasticity of T cell differentiation and cytokine signature: A double-edged sword for immune responses. Immunology, Endocrine \& Metabolic Agents - Medicinal Chemistry (Formerly Current Medicinal Chemistry - Immunology, Endocrine \& Metabolic Agents) 2009; 9:90-105.

[7] Klatt NR, Brenchley JM. Th17 cell dynamics in HIV infection. Curr Opin HIV AIDS 2010; 5:135-40.

[8] Prendergast A, Prado JG, Kang Y, Chen F, Riddell LA, Luzzi G, et al. HIV-1 infection is characterized by profound depletion of CD161+ Th17 cells and gradual decline in regulatory T cells. AIDS 2010;24:491-502 
[9] Yue FY, Merchant A, Kovacs CM, Loutfy M, Persad D, Ostrowski MA. Virus-specific interleukin-17-producing CD4+ $\mathrm{T}$ cells are detectable in early human immunodeficiency virus type 1 infection. J Virol 2008;82:6767-71

[10] Wong H, Pfeiffer RM, Fears TR, Vermeulen R, Ji S, Rabkin CS. Reproducibility and correlations of multiplex cytokine levels in asymptomatic persons. Cancer Epidemiol Biomarkers Prev 2008; 17:3450-56.

Kamat A, Misra V, Cassol E, Ancuta P, Yan Z, Li C, et al. A plasma biomarker signature of immune activation in HIV patients on antiretroviral therapy. PLoS One 2012; e30881.doi:10.1371/journal.pone.0030881. effect of HIV infection and HAART on inflammatory biomarkers in a population-based cohort of US women. AIDS 2011; 25:1823-32.

[13] Rahman S, Connolly JE, Manuel SL, Chehimi J, Montaner LJ, Jain P. Unique Cytokine/Chemokine signatures for HIV-1 and HCV mono-infection versus coinfection as determined by the Luminex® analyses. J Clin Cell Immunol 2011; 2: doi:10.4172/2155-9899.1000104.

[14] Nixon DE, Landay AL. Biomarkers of immune dysfunction in HIV. Curr Opin HIV AIDS 2010; 5:498-503.

[15] Roberts L, Passmore JS, Williamson C, Little F, Bebell LM, Mlisana K, et al. Plasma cytokine levels during acute HIV-1 infection predict HIV disease progression. AIDS 2010; 24:819-31.

[16] Worsley CM, Suchard MS, Stevens WS, Van Rie A, Murdoch DM. Multianalyte profiling of ten cytokines in South African HIV-infected patients with immune reconstitution inflammatory syndrome (IRIS). AIDS Res Ther 2010; 7:36. array can discriminate cytokine secretion from endogenously activated cells in pulmonary disease, recent and remote infection in tuberculosis. Cytokine 2011; $54: 136-43$. 

cytokines as a rapid diagnostic tool for severe infection in paediatric haematology/oncology patients by the use of cytometric bead array technology. Clin Microbio Infect 2011; 17:1666-73.

[19] Tang $\mathrm{Y}, \mathrm{Xu} \mathrm{X}$, Song H, Yang S, Shi S, Wei J, et al. Early diagnostic and prognostic significance of a specific Th1/Th2 cytokine pattern in children with haemophagocytic syndrome. Br J Haematol 2008; 143:84-91.

[20] Frahm M, Goswami ND, Owzar K, Hecker E, Mosher A, Cadogan E, et al. Discriminating between latent and active tuberculosis with multiple biomarker responses. Tuberculosis 2011; 91:250-56.

[21] Hussain R, Kaleem A, Shahid F, Dojki M, Jamil B, Mehmood $H$, et al. Cytokine profiles using whole-blood assays can discriminate between tuberculosis patients and healthy endemic controls in a BCG-vaccinated population. J Immunol Methods 2002; 264:95-108.

[22] Clerici M. Beyond IL-17: New cytokines in the pathogenesis of HIV infection. Curr Opin HIV AIDS 2010; 5:184-88.

[23] Fahey JL. Cytokines, plasma immune activation markers, and clinically relevant surrogate markers in human immunodeficiency virus infection. Clin Diagn Lab Immunol 1998; 5:597-603. Hewer R, Vorster J, Steffens FE, Meyer D. Applying biofluid 1H NMR-based metabonomic techniques to distinguish between HIV-1 positive/AIDS patients on antiretroviral treatment and HIV-1 negative individuals. J Pharm Biomed Anal 2006; $41: 1442-46$.

[25] Jansen CA, De Cuyper IM, Hooibrink B, van der Bij AK, van Baarle D, Miedema F. Prognostic value of HIV-1 gag-specific CD4+ T-cell responses for progression to AIDS analyzed in a prospective cohort study. Blood 2006; 107:142733. 
[26] Brenchley JM, Paiardini M, Knox KS, Asher Al, Cervasi B, Asher TE, et al. Differential Th17 CD4 T-cell depletion in pathogenic and nonpathogenic lentiviral infections. Blood 2008; 112:2826-35.

[27] Khader SA, Gopal R. IL-17 in protective immunity to intracellular pathogens. Virulence 2010; 1:423-27.

[28] Williams A, Koekemoer G, Lindeque Z, Reinecke C, Meyer D. Qualitative serum organic acid profiles of HIV-infected individuals not on antiretroviral treatment. Metabolomics 2011; DOI: 10.1007/s11306-011-0376-2 